- Reports the consensus views of delegates attending the 2005 meeting of the British Association of the Teachers of Conservative Dentistry which addressed the subject of 'Contemporary direct materials and techniques for restoring posterior teeth: Implications for Teaching'.

- Provides guidance for dental educators and teachers of operative/restorative dentistry when placing composite resin restorations in posterior teeth.

- Allows dental educators to compare their existing undergraduate dental educational programmes with the consensus views outlined in this document.

\title{
Teaching posterior composite resin restorations in the United Kingdom and Ireland: consensus views of teachers
}

\author{
C. D. Lynch, ${ }^{1}$ A. C. Shortall, ${ }^{2}$ D. Stewardson, ${ }^{3}$ P. L. Tomson ${ }^{4}$ and F. J. T. Burke ${ }^{5}$
}

Posterior composite resin restorations are an established feature of contemporary dental practice and all new dental graduates should be competent in providing such treatments for their patients. Surveys of educational curricula in this area in the United Kingdom and Ireland, as well as North America, have demonstrated variations both within and between dental schools. Such inconsistency does not help new dental school graduates, and may lead to confusion. At the British Association of Teachers of Conservative Dentistry Annual Conference held in Birmingham in September 2005, a session was devoted to the development of guidelines for dental schools on teaching posterior composite resin restorations to dental undergraduates. The theme of the conference concerned the teaching implications for changing from amalgam to composite. Two of the principal speakers at the meeting (Joost Roeters and Niek Opdam) were from the dental school at the University of Nijmegen in the Netherlands. This school was the first in Europe to discontinue the use of dental amalgam in its undergraduate curriculum over a decade ago. This paper reports the consensus views of those present on guidelines for teaching posterior composite resin restorations to dental undergraduate students.

\section{INTRODUCTION}

The common challenge for dental educators is to train dental practitioners who are competent in treating patients in a general practice setting; an important skill for new dentists being the restoration of posterior teeth that have been damaged by dental caries or trauma. ${ }^{1}$ The key source of information from which many dentists derive guidance and skills

\footnotetext{
"Senior Lecturer/Consultant in Restorative Dentistry, Cardiff University School of Dentistry; ${ }^{2}$ Reader/ Consultant in Restorative Dentistry, University of Birmingham School of Dentistry; 3,4Lecturer/Honorary Specialist Registrar in Restorative Dentistry, University of Birmingham School of Dentistry: ${ }^{5}$ Professor of Primary Dental Care/Consultant in Restorative Dentistry, University of Birmingham School of Dentistry ${ }^{*}$ Correspondence to: Dr Christopher Lynch Email: Iynchcd@cardiff.ac.uk
}

on the use of materials and techniques is the educational and clinical experiences gained at dental school. ${ }^{2}$ In this respect, trends in tooth retention among adults in the United Kingdom and Ireland will mean that, in the future, new dental graduates will be required to manage dentitions containing many restored teeth. ${ }^{3}$ Recent trends in general dental practice have indicated an increase in the placement of direct resin restorations in load-bearing cavities in posterior teeth. ${ }^{4,5}$ The majority of UK general dental practitioners who were surveyed in 2001 were found to place direct composite resin restorations in Class I and Class II cavities of molar teeth. ${ }^{4}$ These findings have been mirrored by the results of a recent investigation that found significantly increased teaching of posterior composite resin restorations in the United Kingdom and Ireland over the last decade. ${ }^{6}$ There was, however, variation and lack of consistency between dental schools on the teaching of aspects of the techniques of placement of posterior composite resin restorations. This inconsistency in teaching has the potential to lead to confusion among new dental graduates. Similar trends were also noted in North American dental schools., ${ }^{7,8}$ The UK and Ireland study commented on the lack of consensus guidelines between dental schools on this subject. ${ }^{6}$ The introduction of such guidelines would be of benefit to all dental schools in the United Kingdom and Ireland in defining educational curricula to appropriately support the professional development of new graduates in this regard.

The British Association of Teachers of Conservative Dentistry (BATCD) is a national organisation which - as its title suggests - represents teachers of Conservative and Restorative Dentistry 
in the United Kingdom and Ireland. It provides support for teachers of conservative/restorative dentistry in their delivery of educational curricula. The BATCD organises an annual conference which addresses contemporary educational challenges in restorative dentistry. In September 2005, in Birmingham, this meeting addressed the topic of composite resin restorations in posterior teeth, with the aim of establishing guidelines on the teaching of posterior composite resin restorations among members. It is hoped that the guidelines outlined will:

i. act as a source in the consideration, development, and harmonisation of educational curricula in dental schools in the United Kingdom and Ireland

ii. be used by teachers and curriculum coordinators of conservative/ restorative dentistry in the United Kingdom and Ireland

iii. be used to develop and harmonise educational criteria in the teaching of posterior composite resin restorations on a larger setting than within the United Kingdom and Ireland

iv. lead to improved quality of care for patients in the United Kingdom and Ireland

v. be used as a basic document and reference against which further educational activities may be benchmarked

vi. be used to inform vocational trainers of guidelines taught to undergraduates and provide a basis for inviting feedback from Vocational Trainees.

The aim of this paper is to outline the consensus views expressed by teachers of conservative/operative dentistry in this area.

\section{METHODS}

A total of 35 teachers contributed to the discussions, divided into five "breakout' groups of six to eight people per group. Each 'breakout' group was given a topic to consider, and then report to the remainder of the meeting. As an initial consideration, it was stressed that educational guidelines in all fields, including posterior composite resin restorations, should be based on best available evidence; however, participants were aware that in certain areas, little or no evidence is available.
The groups considered the teaching of posterior composite resin restorations under the following headings, by way of subheadings, as described below:

- Indications for the use of composite resin

- Cavity design and preparation for posterior composite resin restorations

- The need for, and use of, bases and liners when placing posterior composite resin restorations

- Materials selection and placement technique

- Educational issues surrounding the teaching of posterior composite resin restorations.

A draft copy of this paper was circulated to participants for comment and correction of any errors of fact.

\section{RESULTS AND GUIDELINES}

Indications for the use of composite resin Should amalgam still be taught as a restorative material for the posterior dentition?

It was considered that most dental school curricula have seen an increase in the teaching of posterior composite resin restorations in the last few years, as has already been noted by Lynch et al. ${ }^{6-8}$ This teaching has been at the expense of the teaching of amalgam restorations. It was considered that there were many concerns relating to the use and production of mercury and amalgam. Many dental educators no longer consider amalgam to be the 'material of choice' when restoring posterior teeth. Students are gaining increased exposure to the use of posterior composites, and in some dental schools, students are being taught how to prepare cavities and restore teeth using composites prior to being taught how to do this for amalgam. ${ }^{6-8}$ Within the United Kingdom, however, there is conflict in the sense that the terms of the General Dental Services Act, at the time of writing, provide fees only for placement of amalgam in Class I and Class II cavities, with no fees available for similar composite resin restorations. It is felt that this has limited the expansion of the teaching of posterior composite resin restorations over the last few years. It was suggested at the meeting that, if students are trained how to use composite initially, and continue its clinical use during their course of study, dental schools could aim to move towards a teaching position where a short course could be included in the final clinical year in order to address the educational requirements to achieve competence in the replacement and occasional initial placement of amalgam.

What posterior cavities/situations are indicated for placement of composite?

It was considered that the following are appropriate for posterior composite resin restorations:

- Primary carious lesions including both occlusal and proximal lesions

- Core build-ups

- Restoration of endodontically treated teeth

- Restoration of the worn dentition

- Repair and/or replacement of failed restorations

- Restorations extending onto the root surface may require an alternative layered glass-ionomer cement (or resin-modified glass ionomer cement)/composite resin technique (see 'open-sandwich' technique later in this report).

In all of these, it was considered that success of posterior composite resin restorations is a reflection of the skill of the operator. Achieving effective isolation is essential when placing posterior composites. The rubber dam is considered by some to be a preferred, though not mandatory, means of achieving such isolation. In a situation where proper isolation is not achievable, an alternative restorative material, such as amalgam or glassionomer cement, could be considered.

\section{Management of failed/failing amalgam restorations}

It was considered that a suitable means of managing failed amalgam restorations was to repair the restoration using a composite material, rather than removing the amalgam restoration completely. Following removal of secondary caries, composite could be bonded to the residual tooth structure. It was felt that mechanical retention by way of a dovetail or similar would be required to retain the composite into the remaining amalgam.

\section{In which situations might composite resin materials be unsuitable?}

It was agreed that composite resin materials could be considered for placement in all situations. However, care would 
be advised in the management of elderly patients in whom dentine may be more sclerotic (and perhaps more difficult to bond to). Caution was also advised in the placement of composite onlays in a bruxist who was unable to comply with biteguard therapy.

\section{Isolation}

Proper isolation for posterior composite resin restorations is essential (though not necessarily with rubber dam). If this is not possible, then the tooth should be restored on a temporary basis using glass-ionomer cement, and isolation reattempted at a later time.

\section{Cavity design and preparation for posterior composite resin restorations \\ Timing of intervention}

Dental caries was considered as a disease process that may be prevented, or in its early stages, reversed by appropriate oral hygiene practices. Diagnosis should be based on clinical and radiographic features, and teeth should be examined when air-dried and using proper illumination. It was considered important to remember that when a lesion is observed radiographically, it may sometimes be arrested. Correlation of radiographic features with clinical features is always indicated. The indication for restoration of a carious lesion is a cavity that cannot be rendered plaque free on a routine basis by the patient. ${ }^{9}$

It was felt that the placement of composite resin was more advantageous in smaller cavities than amalgam, as less healthy tooth tissue removal is required during cavity preparation.

\section{Caries removal}

It is important that, following caries removal, the amelo-dentinal junction should feel hard to tactile sensation with a dental explorer although it does not need to be rendered stain free. It should be remembered, however, that the presence of stained dentine at the amelo-dentinal junction may give a discoloured appearance to the tooth. The pulpal floor could be considered cariesfree when it feels hard to tactile sensation with a dental excavator. In keeping with contemporary evidence, it was not considered necessary to always remove soft stained dentine from the floor of the cavity. Indeed, there is little evidence that infected dentine must be removed before sealing the lesion with the adhesive composite restoration. ${ }^{9}$ However, it was acknowledged that there was a substantial amount of research being undertaken in this area, and that teaching should take account of the results of this research when it becomes available.

\section{What should the outline form for posterior composite resin restorations be?}

The outline form for a posterior composite resin restoration will be determined by the size and the extent of the caries. Removal of additional tooth tissue may be indicated to facilitate access for instrumentation. Undercuts are not necessary for posterior composite resin restorations. However, if these are present following caries removal, there is no need to remove them.

Bevelling of the occlusal margins was considered to be contraindicated, as this may lead to fracture of the resulting thin extensions of composite resin material. ${ }^{10}$ Furthermore, it was considered that bevelling of the occlusal margin may lead to extension of the cavity when future replacement or refurbishment of the restoration is required.

Bevelling of the margins of the proximal box is generally indicated, except when there is little remaining enamel bulk cervically, or if restoration margins finish on dentine or cementum.

There was no clear consensus to support bevelling of the occluso-axial line angle in cavity preparations.

\section{Tunnel preparations}

Concern was expressed regarding the use of tunnel preparations. The reasons for this include reduced access for visual inspection, reduced access for placement of the restoration, and the risk of subsequent fracture of the overlying marginal ridge. ${ }^{11}$

The need for, and use of, bases and liners when placing posterior composite resin restorations

It was recognised that there is now a wide range of commercially available products and techniques for bonding or basing so-called 'deeper' cavities. Furthermore, as there is a lack of consensus among the research community as to which is the most suitable or 'best' technique, this is often reflected in the variations in teaching on this subject. Some clinicians prefer to apply a base to cavities extending into dentine; others prefer to use a 'total-etch' approach. However, participants agreed that the end-goal of both techniques should be that the margins of the final restoration are adequately sealed to prevent leakage. When using any form of material, including bonding agents or bases, it is essential that the manufacturers' instructions are followed closely.

If a pulp-capping agent such as fastsetting calcium hydroxide is applied to the base of the cavity, participants felt that it is advisable that a thin layer of resin-modified glass-ionomer cement is applied over this for protection, prior to etching and placing the composite restoration.

It is suggested that for routine bonding applications such as in a 'non-retentive' cavity, which has little or no mechanical retention following removal of previous restorative materials and/or caries removal, that a three-step total etch approach (ie separate etchant, primer, and bonding agent) be used as there is clear research evidence to support this. ${ }^{12}$ In cavities that have undercuts and some degree of retention is already present, or where there is a risk of moisture control being compromised by rinsing after etching, it may be advantageous to use a two-step or self-etching bonding technique, as there is good long term clinical data supporting the use of at least one of these systems. ${ }^{12}$ They are considered by many to be less technique sensitive and thereby associated with less post-operative sensitivity. When dealing with sclerotic dentine, it may be advantageous to lightly abrade the dentine surface prior to application of the bonding agent and possibly also to place a cervical retention groove.

There was a lack of clear evidence surrounding the use of the 'sandwich technique'. A closed sandwich technique may be indicated where achieving adequate moisture control is a concern, such as an uncooperative patient. This allows restoration of the portion of the cavity that extends into dentine with glass-ionomer (or resin-modified glass-ionomer) cement to the level of the amelo-dentinal junction. The enamel is etched and the remaining portion of the cavity is restored with composite. Unpublished data $^{13}$ have demonstrated a higher incidence of bulk fracture in load bearing posterior composite restorations restored 
with a closed sandwich technique in comparison to dentine bonded composite control restorations over a five year period. An open sandwich technique may be appropriate when restoring a cavity that extends subgingivally, especially when the patient has a high caries rate. In such situations, the portion of the cavity extending onto root dentine and cementum may be restored with resin-modified glass-ionomer cement, prior to the application of the composite material. Encouraging clinical trials data (low incidence of recurrent caries and relatively low fracture rates) up to five years' duration have been reported for this technique.

\section{Placement techniques and \\ material selection}

Choice of composite material

It was recognised that there are different physical requirements for composite materials placed in either anterior or posterior cavities, as reported by Roeters et al. ${ }^{14}$ While composite materials for anterior cavities may sacrifice certain functional properties for aesthetic concerns, the converse should be true for composite materials in posterior cavities. Composite materials for posterior cavities should be of appropriate compressive strength to support occlusal function, even if this means compromising aesthetic properties.

It may be advantageous that the shade of material selected for restoration of the posterior cavity is a slightly lighter shade to the tooth to allow differentiation of the composite material should the restoration need to be removed at a later time.

\section{Selection of matrix bands and proximal wedges}

It was considered that the use of metal (either sectional or circumferential) matrix bands and wooden wedges are associated with more favourable outcomes than transparent matrix bands and light-transmitting wedges, as reported by Mullejans et al. ${ }^{15}$ Pre-contoured metal matrices in association with a 'layered' curing technique were considered to facilitate creation of a proper proximal contact.

\section{Isolation}

It is essential that proper isolation be achieved when placing posterior composites. Rubber dam is helpful for this purpose, though not always required. It was considered that posterior composite restorations placed using effective cotton wool roll/matrix band isolation may achieve similar survival rates to those placed using rubber dam isolation, this consideration being in agreement with the work of Brunthaler et al. ${ }^{16}$

\section{Curing lights}

Current evidence suggests that the current high power 'second generation' LED light activation lights produce comparable depth of cure and composite properties for the vast majority of (Camphorquinone initiated) composites in comparison to 'traditional' quartztungsten-halogen curing lights in the same or less radiation time. ${ }^{17,18}$ Whether the more recently introduced "third generation' dual peak (violet and blue LEDs) wavelength LED units will match their high power QTH counterparts has yet to be established. The ability to reduce exposure times without compromising restoration quality will encourage the more widespread uptake by practitioners of adhesive restorative solutions. Student exposure to new techniques and technologies should always be encouraged, as this will encourage their familiarisation with commercial products, and reduce their confusion during subsequent independent practice.

\section{Finishing techniques}

A variety of products are commercially available for this purpose, such as discs, polishing burs, and interproximal polishing strips. Product selection is often a matter of clinical preference, but it is important that whatever meticulous technique is used, smooth surfaces with excellent marginal adaptation, particularly proximal and cervical surfaces, are produced.

Educational issues surrounding the teaching of posterior composite resin restorations It was recognised that, in light of the increased use of composite materials in posterior teeth in dental practice, that there were increased demands on dental educators and clinical teachers to ensure that their students gain appropriate didactic and clinical exposure to the placement of composite in posterior teeth. However, there is much variation between dental schools in the United Kingdom and Ireland on how this may be achieved, some schools having limited teaching of posterior composites, while other schools have more extensive teaching. The possible reasons behind the current situation and challenges facing teaching of posterior composites in the United Kingdom and Ireland has been highlighted in a recent paper. ${ }^{19}$

Effective teaching of posterior composite resin restorations is compromised by:

- The amount of curriculum time available

- Variations of teaching between clinical teachers

- GDS regulations did not, at the time of writing, allow placement of composite in Class I or Class II cavities, much teaching is now undertaken in outreach centres where GDS Regulations apply

- Cost of materials and necessary equipment such as matrices, curing lights, etc

- Due to need for adequate moisture control, close support is required from dental nurses; such staff resources may not always be available.

\section{CONCLUSION}

This report has described the conclusions of the recent British Association of Teachers of Conservative Dentistry consensus meeting on posterior composite resin restorations. It was evident from discussions among the group that there were variations both between and within dental schools on the teaching of this topic. Such inconsistencies may lead to confusion among new dental graduates; these should be addressed in future curriculum development. It is hoped that this article will provide support for dental educators in this task.

The authors wish to express their gratitude to those of their colleagues, too many to acknowledge individually, who contributed to the discussions and made for a very successful meeting. In particular we wish to express our thanks to Joost Roeters and Niek Opdam for giving so generously of their time to support the conference and for taking a leading role internationally in this field.

1. Plasschaert A J M, Holbrook W P, Delap E et al. Profile and competences for the European Dentist. Eur J Dent Educ 2005: 9: 98-107.

2. Wilson N H F, Dunne S M, Gainsford I D. Current materials and techniques for direct restorations in posterior teeth. Part 2: resin composite systems. Int Dent J 1997; 47: 185-193.

3. Kelly M, Steele J, Nuttall N et al. Adult Dental Health Survey - oral health in the United Kingdom 1998. London: The Stationery Office, 2000.

4. Burke F J T, McHugh S, Hall A C et al. Amalgam and composite use in UK general dental practice in 2001. Br Dent J 2003; 194: 613-618.

5. Brown $L J$, Wall T, Wassenaar J D. Trends in resin and amalgam usage as recorded on insurance 
claims submitted by dentists from the early 1990s and 1998. J Dent Res 2000; 79: 461.

6. Lynch C D, McConnell R J, Wilson N H F. Teaching of posterior composite resin restorations in undergraduate dental schools in Ireland and the United Kingdom. Eur J Dent Educ 2006; 10: 38-43.

7. Lynch C D, McConnell R J, Wilson N H F. Teaching posterior composites in US dental schools. J Am Dent Assoc 2006: 137: 619-625.

8. Lynch C D, McConnell R J, Wilson N H F. Teaching the use of posterior resin composites in Canadian dental schools: how do current educational practices compare with North American trends? J Can Dent Assoc 2006; 72: 321.

9. Kidd E A M. How 'clean' must a cavity be before restoration? Caries Res 2004; 38: 305-313.

10. Wilson N H F, Wilson M A, Wastell D G et al.
Performance of Occlusin in butt-joint and beveledged preparations: five year results. Dent Mater 1991; 7: 92-98.

11. Ratledge D K, Kidd E A, Treasure E T. The tunnel restoration. Br Dent J 2002; 193: 501-506.

12. Peumans M, Kanumilli P, De Munck J et al. Clinical effectiveness of contemporary adhesives: a systematic review of current clinical trials. Dent Mater 2005; 21: 864-881.

13. Opdam N J M, Bronkhorst E M, Roeters J J M, Loomans B A C. Longevity and reasons for failure of sandwich and total-etch posterior composite resin restorations. J Adhes Dent: in press.

14. Roeters J J M, Shortall A C C, Opdam N J M. Can a single composite resin serve all purposes? BrDent J 2005; 19: 73-79.

15. Mullejans R, Badawi M O, Raab W H et al.
An in vitro comparison of metal and transparent matrices used for bonded Class II resin composite restorations. Oper Dent 2003; 28: 122-126.

16. Brunthaler A, Konig F, Lucas T et al. Longevity of direct resin composite restorations in posterior teeth. Clin Oral Investig 2003; 7: 63-70.

17. Price R B, Felix C A, Andreou P. Knoop hardness of ten resin composites irradiated with high-power LED and quartz-tungsten-halogen lights. Biomaterials 2005; 26: 2631-2634.

18. Shortall A C. How light source and product shade influence cure depth for a contemporary composite. J Oral Rehabil 2005; 32: 906-911.

19. Lynch C D, McConnell R J, Wilson N H F. Challenges to teaching posterior composites in the United Kingdom and Ireland. Br Dent J 2006; 201: 747-750. 